\title{
FreeHi-C: high fidelity Hi-C data simulation for benchmarking and data augmentation
}

\author{
Ye Zheng ${ }^{1} \&$ Sündüz Keleş ${ }^{1,2, *}$ \\ ${ }^{1}$ Department of Statistics, University of Wisconsin - Madison, Madison, WI 53706, USA \\ ${ }^{2}$ Department of Biostatistics and Medical Informatics, University of Wisconsin - \\ Madison, Madison, WI 53706, USA \\ * Corresponding author
}

\begin{abstract}
Ability to simulate realistic high-throughput chromatin conformation (Hi-C) data is foundational for developing and benchmarking statistical and computational methods for Hi-C data analysis. We propose FreeHi-C, a data-driven $\mathrm{Hi}-\mathrm{C}$ simulator for simulating and augmenting $\mathrm{Hi}-\mathrm{C}$ datasets. FreeHi-C employs a non-parametric strategy for estimating interaction distribution of genome fragments from a given sample and simulates $\mathrm{Hi}-\mathrm{C}$ reads from interacting fragments. Data from FreeHi-C exhibit higher fidelity to the biological Hi-C data compared with other tools in its class. FreeHi-C not only enables benchmarking a wide range of Hi-C analysis methods but also boosts the precision and power of differential chromatin interaction detection methods while preserving false discovery rate control through data augmentation.
\end{abstract}

\section{Introduction}

Recent maturation of chromosome conformation capture (3C) $)^{11}$ and Hi-C sequencing technologies 23 led to high-throughput profiling of three-dimensional chromatin architecture and revealed transformative insights on long-range gene regulation ${ }^{4}$. Alongside the technological breakthroughs, a growing number of methodologies and algorithms ${ }^{7-16}$ emerged for the analysis of Hi-C and other 3C-derived data types. These methods are developed and benchmarked on disparate biological and simulated or computationally-constructed datasets that are often customized for the methods under the study. Furthermore, the high sequencing costs of Hi-C experiments, e.g., billions of reads for decent $10-40 \mathrm{~kb}$ analysis resolution of mammalian genomes ${ }^{317}$, preclude the possibility of generating biological benchmarking datasets with sufficient sequencing depth, numbers of replicates, and with known true interactions.

Benchmarking and evaluation of $\mathrm{Hi}-\mathrm{C}$ methods have relied on either conducting distancestratified permutations of the contact matrices ${ }^{\sqrt{15}}$ to nullify the underlying biological structure or simulating contact matrices directly by capturing only the most general spatial structures

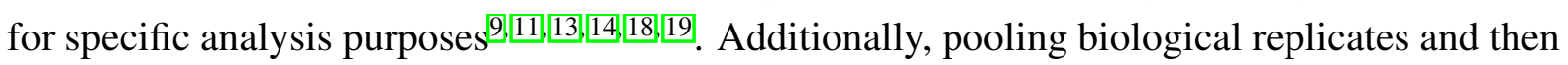
randomly partitioning to generate pseudo-replicates and downsampling are also common approaches to study similarity metrics or evaluate the sequencing depth effect ${ }^{12} 151819$. A more systematic Hi-C simulation method, $\operatorname{Sim} 3 \mathrm{C}^{\sqrt{20}}$, has been recently proposed for the design of Hi$\mathrm{C}$ experiments with respect to the selection of restriction enzyme, determination of sequencing depth, and power analysis. However, Sim3C imposes strong assumptions to parametrically mimic $\mathrm{Hi}-\mathrm{C}$ contact matrix structures and arbitrarily introduces random domain structures, e.g., topological associating domains (TADs). The properties of actual chromatin interactions at dif- 
bioRxiv preprint doi: https://doi.org/10.1101/629923; this version posted May 14, 2019. The copyright holder for this preprint (which was not certified by peer review) is the author/funder, who has granted bioRxiv a license to display the preprint in perpetuity. It is made available under aCC-BY-NC-ND 4.0 International license.

ferent scales $\frac{1121-[23}{23}$ are far more nuanced than the basic factors that Sim3C takes into account. Consequently, the resulting Sim3C simulated contact matrices do not visually resemble biological Hi-C contact matrices (Fig. 117).

\section{Results}

In this paper, we present a robust Hi-C simulation method, FreeHi-C (Fragment interactions empirical estimation for fast simulation of $\mathbf{H i}-\mathbf{C}$ data) that simulates realistic read-level Hi-C data leveraging a nonparametric framework. FreeHi-C takes as input raw $\mathrm{Hi}-\mathrm{C}$ sequencing reads in FASTQ format and estimates the frequency of genomic fragment interactions. This is fundamentally different from existing methods that simulate $\mathrm{Hi}-\mathrm{C}$ contact matrices under a series of assumptions 91114 15. Subsequently, FreeHi-C generates pairs of sequencing reads that represent the interacting fragment pairs with embedded random nucleotide mutations and indels while conserving the proportion of chimeric reads. Thereby, the variability and readlevel characteristics of original Hi-C sequencing libraries can be preserved in the simulated sequences. Overall, the entire simulation procedure of FreeHi-C emulates the standard Hi-C experimental protocol ${ }^{23}$ (Fig. 1 1 ) where each read pair is generated independently, thereby allowing parallelization for faster implementation and the ability to simulate to the user-specified sequencing depth. The ability to simulate larger depths enables performance assessment of $\mathrm{Hi}-\mathrm{C}$ analysis methods such as significant interaction and differential interaction detection with respect to varying sequencing depths. The method can be used to simulate Hi-C data from any organism, with any read length, and restriction enzymes. FreeHi-C is freely available at https: / / github.com/keleslab/freehic and includes a complete standard Hi-C data processing module ${ }^{824}$ encompassing raw sequence alignment, validation checking, duplicate removal, and genome binning. Hence the output is compatible with the standard input of downstream analysis such as normalization. Simulation component is an order of magnitude less time consuming than processing of the sequencing reads (Supplementary Fig. 1).

We illustrated the versatile features of FreeHi-C with Hi-C datasets of two human cell lines, GM12878 and A549, and malaria parasite Plasmodium falciparum 3D7, as representatives of large and small genomes, respectively. Analysis of GM12878 and A549 are carried out at $40 \mathrm{~kb}$ resolution and $10 \mathrm{~kb}$ for Plasmodium falciparum 3D7. Replicates are simulated at the same sequencing depth as the original biological replicates unless explicitly stated. For illustration purposes, we represent the analysis results utilizing all the interactions involving chromosome 1 of biological replicates, rep2, rep3, rep4, and rep6, for GM12878 and rep1 to rep4 for A549 cell line and all the 14 chromosomes from three life stages of $P$. falciparum. The sequencing depths of the replicates are summarized in Supplementary Fig. 2 .

We first assessed how well FreeHi-C simulated replicates capture the general data characteristics of the biological replicates. Juicebox ${ }^{25}$ visualizations of the contact matrices of a FreeHi-C simulated dataset compared to the seed biological replicate and Sim3C realization with matching parameters are displayed in Fig. 117. FreeHi-C simulated contact matrix exhibits markedly higher fidelity to the original biological replicate. Furthermore, the local interaction 
bioRxiv preprint doi: https://doi.org/10.1101/629923; this version posted May 14,2019. The copyright holder for this preprint (which was not certified by peer review) is the author/funder, who has granted bioRxiv a license to display the preprint in perpetuity. It is made available under aCC-BY-NC-ND 4.0 International license.

visualization in Supplementary Figs. 3.5 5 illustrates that FreeHi-C robustly captures the detailed chromatin interaction structures, such as the chromatin loops and TADs, for all biological replicates at any genomic location. Next, comparison of the distribution of contact counts stratified by the genomic distance between the simulated and biological replicates shows that simulated reads of the original sequencing depth give rise to contact count distributions similar to those of the seed biological replicates (Supplementary Figs. 6 and 7). After establishing high fidelity of individual simulation replicates, we assessed whether the simulated data of different biological replicates preserved the relationships existing between biological replicates. Fig. 11 displays hierarchical clustering of three biological $P$. falciparium samples together with three FreeHi-C simulated replicates each. The high fidelity of simulations visualized in Supplementary Fig. 8 leads to each simulated replicate being clustered with its seed biological sample and, furthermore, retains the relationship that chromatin architecture in Rings stage is more similar to that of Schizonts stage while Trophozoites stage has striking differences from the other two ${ }^{26}$. In addition to recapitulating the clustering structure of the biological samples, when compared in pairs, simulated replicates also display similar distance stratified log-fold-change trends of the contact counts as the biological seed samples (Supplementary Figs. 9 and 10).

Next, we demonstrate how FreeHi-C enables benchmarking a wide range of Hi-C analysis methods and compare it with the commonly used downsampling approach. Our first analysis focuses on the assessment of the reproducibility of Hi-C contact matrices by HiCRep ${ }^{12}$. Although Fig. 1; successfully clustered Rings and Schizonts stages together based on the HiCRep reproducibility quantification, this result can be challenged as biased due to the significant differences in the sequencing depths of the samples, i.e., Rings and Schizonts stages have 45$375 \%$ more reads (Supplementary Fig. 2). Therefore, it is desirable to adjust for the sequencing depth either by simulation or downsampling and re-evaluate the performance of HiCRep. Fig. 1 $1 \mathrm{~d}$ and Supplementary Fig. 11 illustrate that by down-simulating Rings and Schizonts stages to the smallest sequencing depth sample Trophozoites or up-simulating Trophozoites and Rings stages to that of the largest sample Schizonts, HiCRep reproducibility can consistently cluster Rings and Schizonts stages together. Downsampling, however, leads to Schizonts being mis-clustered with Trophozoites (Fig. 1) indicating that downsampling may generate low-quality Hi-C matrices (i.e., reproducibility of Hi-C matrices from downsampled datasets ranged between 0.32 and 0.91 ) that potentially lead to wrong inference. To further explore the impact of removing sequencing depth bias on HiCRep reproducibility, we applied HiCRep between biological and the corresponding simulated replicates of GM12878 data. Supplementary Fig. 12. replicates increases with the growing sequencing depths. However, we observe that the deeply sequenced biological replicates, e.g., rep2 and rep3 of GM12878, can achieve as high reproducibility with the smaller biological replicates as the low sequenced simulated replicates of rep4 and rep6 (Supplementary Fig. 12a). This contradicts the expectation that reproducibility of a biological replicate with another independent biological replicate cannot exceed the reproducibility with its FreeHi-C simulated replicate. By simulating the biological replicates, for 
example, rep2, rep3, and rep6, to the sequencing depth of the target sample rep4, we eliminate the sequencing depth bias and unveil the underlying similarity relationship (Supplementary Fig. 12p). In contrast, downsampling strategy can only be implemented for the smallest replicate, namely downsampling rep2 to rep4 to sequencing depth of rep6 (Fig. 11). Hence, FreeHi-C facilitates evaluation of HiCRep and similar methods at a wider range of sequencing depths without any limitation (Fig. 1 $\mathrm{g}$ ). We provide a similar sequencing depth debiasing discussion for Fit-Hi- $\mathrm{C}^{7}$ in detecting significant interactions in Supplementary Materials.

Another pivotal benchmarking utility of FreeHi-C is for comparing performances of different methods that address the same Hi-C inference problem. We considered the detection of differential chromatin interactions (DCIs) as an example and compared the state-of-the-art differential interaction identification methods, diffHic ${ }^{9}$ and multiHiCcompare ${ }^{16}$, across a series of sequencing depths. We singled out these two approaches because alternatives such as FIND and HiCcompare ${ }^{\sqrt{14}}$ have either established lack of false discovery rate control and excessively long run times ${ }^{1419}$ or constituted a specialized version of multiHiCcompare to exclusively handle one replicate per condition. We compared diffHic and multiHiCcompare with respect to false discovery rate (FDR) control and power through both FreeHi-C simulation and downsampling. Overall, Supplementary Fig. 13 elucidates that both methods can control the FDR when replicates are evaluated for DCIs within the GM12878 data. Remarkably, downsampling displays an increasing FDR trend as the sequencing depth increases; however, this trend can only be investigated up until the original depth of the samples since the sequencing depths of downsampled samples cannot go beyond their original depths. FreeHi-C simulation elucidates the trends when the depths of the samples go beyond their original ones and illustrates conservative FDR control at higher depths. This highlights a clear pitfall of drawing conclusions solely based on downsampling. Additionally, downsampling and FreeHi-C simulation have distinct implications for detection power of the methods (the first column of Supplementary Fig. 14). FreeHi-C consistently indicates higher detection power for multiHiCcompare than diffHic; however, downsampling reverses the rank of the two methods and only reveals the same conclusion as FreeHi-C after small log-fold-change interactions are filtered (the second and third columns of Supplementary Fig. 14). Collectively, FreeHi-C assesses performances of competing methods without any sequencing depth limit. Downsampling, however, can only accommodate comparisons at lower sequencing depths of the available samples and may lead to inconsistent or unreliable discoveries.

A key impediment for differential chromatin interaction inference with $\mathrm{Hi}-\mathrm{C}$ data is the limited numbers of biological replicates. As a result, numerous practical challenges arise. We identified three commonly encountered scenarios as a function of the number of replicates available per condition (Fig. 2) and assessed how FreeHi-C simulated replicates can offer advancements. These three settings are one replicate per condition (ORPC), uneven numbers of replicates per condition where one of the conditions have only a single replicate (URPC), and multiple numbers of replicates per condition (MRPC). Under these settings, we applied multiHiCcompare which demonstrated stronger power in the previous analysis, for differential 
bioRxiv preprint doi: https://doi.org/10.1101/629923; this version posted May 14,2019. The copyright holder for this preprint (which was not certified by peer review) is the author/funder, who has granted bioRxiv a license to display the preprint in perpetuity. It is made available under aCC-BY-NC-ND 4.0 International license.

chromatin interaction detection. For all the settings, we first provide the assessment that established false discovery rate control and then investigate detection power and the precision of top significant findings, where the set of differential interactions from the full set of biological replicates (4 from each of the A549 and GM12878 cells) is utilized as the gold standard. In this first setting ORPC where each condition has only one replicate, the false discovery rate control with the $\mathrm{BH}$ procedure ${ }^{27}$ ensures that the observed FDR is below the target FDR (the first panel in Fig. 2a and Supplementary Fig. 15); however, overall, this setting exhibits extremely conservative FDR control. This comes, not so unexpectedly, at the cost of low power (the third panel in Supplementary Fig. 16. When we augment each of the conditions with FreeHi-C simulated replicates, FDR is still well controlled (Fig. 2a and Supplementary Fig. 15), and power increases by an average of 300 folds across the five levels of FDR thresholds, going from only a few hundred differential interactions to a few tens of thousands (Supplementary Fig. 16p). It is reasonable to argue that under ORPC, the key target should not be a discrete, thresholded list of DCIs. Instead, the focus should be on the ranking of the interactions because the top significant DCIs are typically utilized for downstream analysis and experimental follow-up. We scrutinized the accuracy of the top significant DCIs identified with and without FreeHi-C augmentation by comparing the ranked lists to the set of DCIs identified by utilizing all the 4 biological replicates of the two conditions. Fig. 2p illustrates that FreeHi-C simulated samples lead to a significantly higher precision of $100-75 \%$ compared to $\sim 10 \%$ of the ORPC analysis (evaluations under additional gold standard settings are provided in Supplementary Fig. 17). We next evaluated the biological relevance of the "ranked up" and "ranked down" DCIs due to FreeHi-C augmentation by external RNA-seq and CTCF ChIP-seq data. The new ranking of the top DCIs overlapped significantly more with the differentially expressed genes of the two cell lines (Supplementary Fig. 18a), supporting FreeHiC simulation augmentation. Overlap with differential CTCF ChIP-seq peaks deemed both the original and the new rankings similarly significant (Supplementary Fig. 18p) except for a clear advantage for ranking with the FreeHiC augmentation at the very top 100 DCIs. An immediate extension of the ORPC includes uneven numbers of replicates per condition, particularly, single replicate for one and two or three replicates for the other. URPC may arise either by design or due to low quality or failed replicates. Figs. 2 2 and d for URPC highlight that, similar to ORPC, FreeHi-C augmentation of the conditions markedly boosts power (Supplementary Fig. 19p), refines ranking of the DCIs (Supplementary Figs. 20.22) while preserving FDR control (Supplementary Fig. 19a), and exhibiting strong genomic support (Supplementary Fig. 23).

Finally, we generalized the FreeHiC augmentation strategy as a meta-analysis approach for the general setting of multiple replicates per condition (Figs. 2 2 and f). To account for the fact that simulated samples cannot provide additional full degrees of freedom, the meta-analysis approach pairs biological and simulated samples in numbers concordant with the original differential testing design and aggregates p-values of candidate differential interactions across comparisons by Fisher's method ${ }^{28}$. It then refines the significance ranking for FDR control with the $\mathrm{BH}$ procedure by taking the median of the adjusted p-values per interaction (Meth- 
bioRxiv preprint doi: https://doi.org/10.1101/629923; this version posted May 14,2019. The copyright holder for this preprint (which was not certified by peer review) is the author/funder, who has granted bioRxiv a license to display the preprint in perpetuity. It is made available under aCC-BY-NC-ND 4.0 International license.

ods). Fig. 22 and Supplementary Fig. 24 reveal that FDR control is well preserved as the number of simulated replicates in augmentation increases. Notably, augmentation with simulated replicates not only boosts the number of significant DCIs identified across a series of FDR thresholds (Supplementary Fig. 25), but also results in a significantly better ranking with higher precision of the top significant DCIs for further quantitative and experimental validation (Fig. 2f and Supplementary Figs. 26,31). We further evaluated the biological relevance of the DCIs identified with meta-analysis via FreeHiC augmentation by leveraging RNA-seq and CTCF ChIP-seq data. With the enrichment levels using the full set of 4 replicates per condition serving as the best achievable outcome (panel b of Supplementary Figs. 32 and 33, meta-analysis via FreeHi-C augmentation successfully improves the significance of the overlapping between DCIs and the differentially expressed genes (Supplementary Figs. 32 and 33 for MRPC with 3 and 2 replicates per condition, respectively). Evaluation with differential CTCF ChIP-seq peaks results in significant co-localization for all the detection settings with or without FreeHiC augmentation (Supplementary Fig. 34).

Analytical methods for analyzing data from $\mathrm{Hi}-\mathrm{C}$ and related experiments are growing at a faster pace than they are benchmarked and evaluated uniformly. We developed FreeHi$\mathrm{C}$ to enable simulating read-level Hi-C data in a data-driven manner. A direct advantage of FreeHi-C non-parametric construction by closely following the Hi-C experimental protocol is that contact matrices resulting from FreeHi-C simulated reads accurately capture the nuanced interaction structures of real Hi-C contact matrices. We showcased the benchmarking utility of FreeHi-C simulations by investigating the performance of HiCRep ${ }^{12}$ for reproducibility quantification, Fit-Hi-C $\mathrm{C}^{7}$ for significant interaction detection, and diffHic ${ }^{\sqrt{9}}$ and multiHiCcompare ${ }^{\sqrt{16}}$ for identifying DCIs. We demonstrated the outperformance of FreeHi-C over the downsampling approach with respect to flexibility and reliability as a benchmarking tool. Most notably, we illustrated that data augmentation with FreeHi-C simulation improves detection of differential chromatin interactions across all the settings parametrized by the numbers of available replicates per condition while preserving FDR control. FreeHi-C augmentation led to markedly raises the accuracy of ranked DCIs with better biological support compared to analysis only with the original replicates. The robust performance of FreeHi-C data augmentation leads to more accurate lists of DCIs which are essential for effective downstream analysis.

Acknowledgments This work was supported by NIH grants HG009744 and HG007019 to Sündüz Keleş.

Author Contributions S.K. and Y.Z. conceived the project. Y.Z. and S.K. designed the research and developed the method. Y.Z. developed the simulation framework and performed the experiments. Both authors contributed to the preparation of the manuscript.

Competing Interests The authors declare no competing financial interests.

Correspondence Sündüz Keleş (keles@stat.wisc.edu). 
bioRxiv preprint doi: https://doi.org/10.1101/629923; this version posted May 14,2019. The copyright holder for this preprint (which was not certified by peer review) is the author/funder, who has granted bioRxiv a license to display the preprint in perpetuity. It is made available under aCC-BY-NC-ND 4.0 International license.

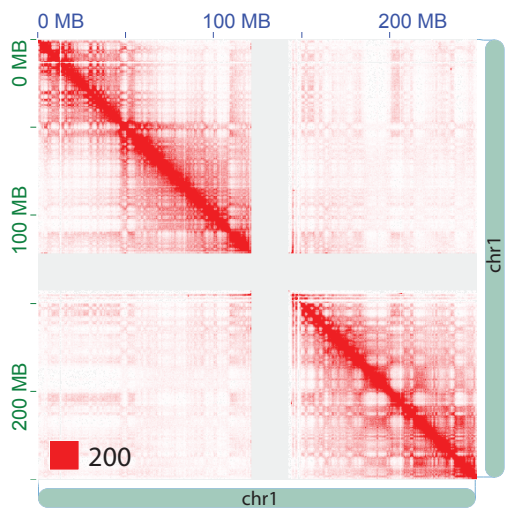

FreeHi-C Simulated Replicate

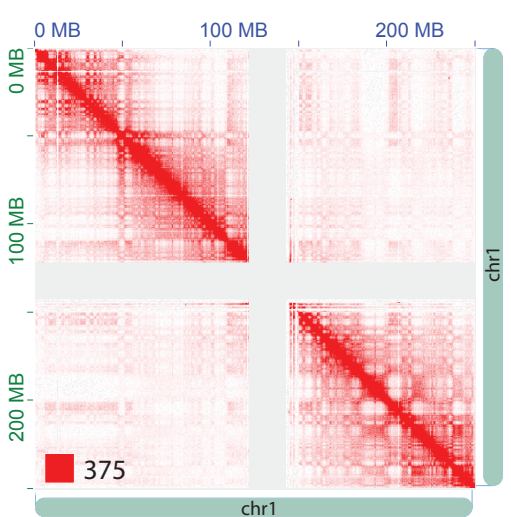

Sim3C Simulated Replicate

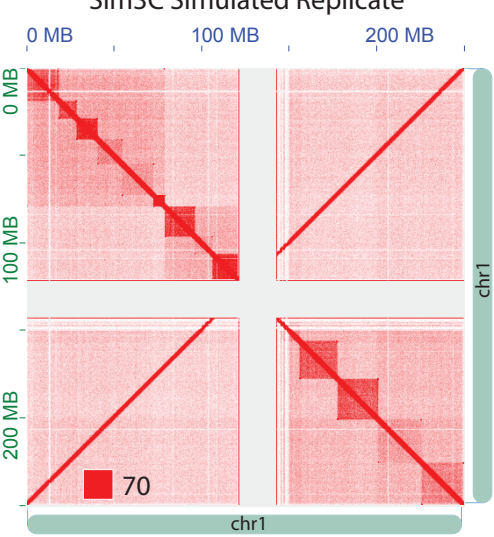

b

\begin{tabular}{|l|l|}
\multicolumn{1}{|c|}{ Step 1 } \\
\hline Hi-C sequencing data: \\
Sample_end1.fastq \\
Sample_end2.fastq
\end{tabular}$\longrightarrow$\begin{tabular}{l} 
Processing and training \\
- Alignment \\
- Read ends pairing \\
- Validation checking \\
- Duplicates removal \\
- Binning \\
\hline
\end{tabular}

Step 2
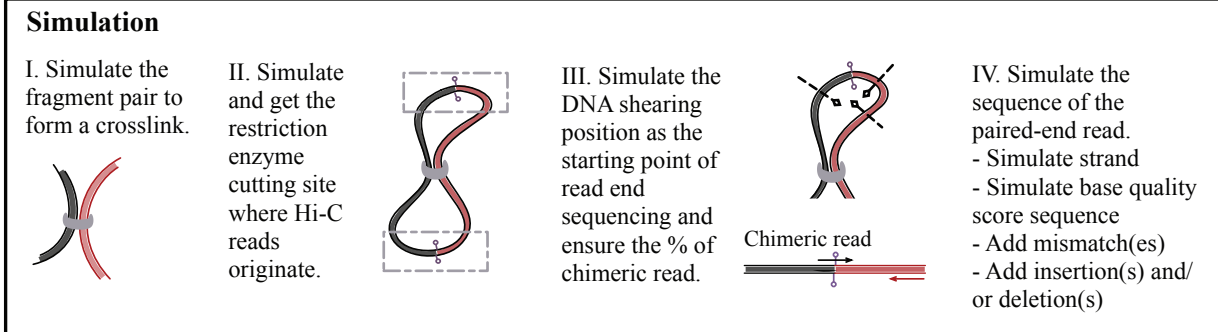

Training output

1. Frequency of fragment interactions

2. \% of mismatch(es), insertion(s), and deletion(s)

3. Distribution of mismatch(es), insertion(s), and deletion(s)

4. $\%$ of chimeric reads

5. Distribution of base quality scores

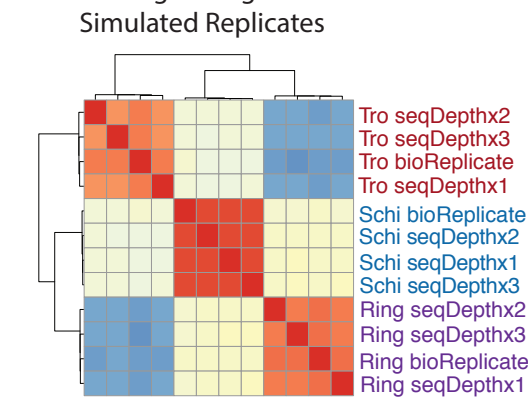

d Clustering of Downsimulated Replicates

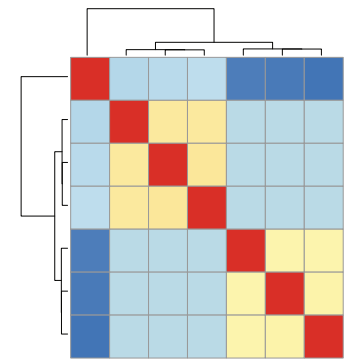

Tro BioReplicate Schi Downsimu2 Schi Downsimu1 Schi Downsimu3 Ring Downsimu2 Ring Downsimu1 Ring Downsimu3

\section{Simulation output}

Hi-C simulated sequencing data: Simulation_end1.fastq Simulation_end2.fastq

\begin{tabular}{|l|} 
Step 3 \\
Simulated reads processing \\
- Alignment \\
- Read ends pairing \\
- Validation checking \\
- Binning
\end{tabular}

f

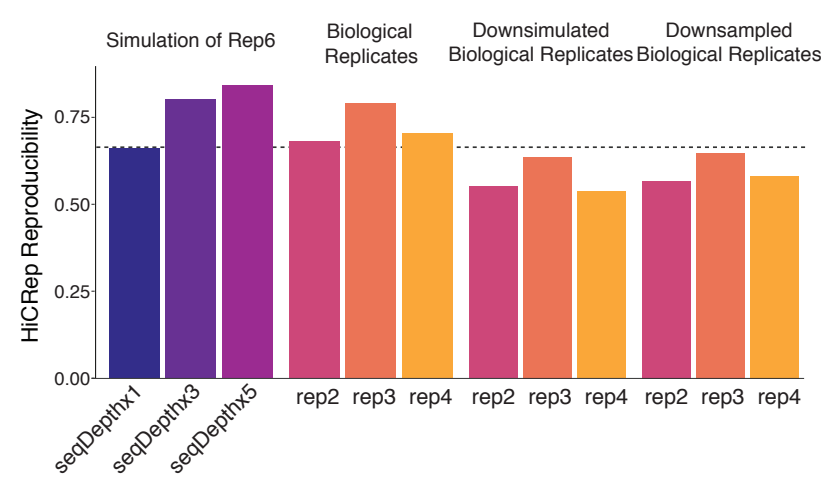

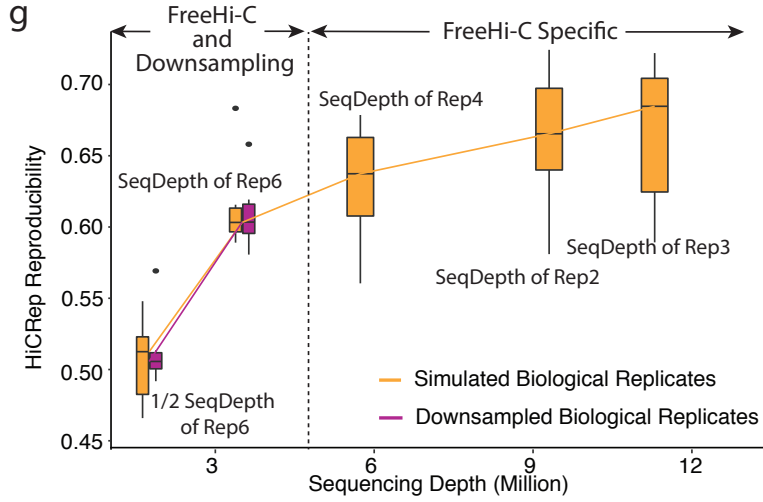


Figure 1 FreeHi-C enables simulating high fidelity $\mathrm{Hi}-\mathrm{C}$ data. a. Hi-C contact matrix of biological rep2 of GM12878 along with FreeHi-C and $\mathrm{Sim}^{2} \mathrm{C}^{20}$ simulated replicates at matching sequencing depths. FreeHi-C and Sim3C parameters are set based on rep2. The numbers at the left bottom of the matrices are the color scale for the interaction counts. b. Details of the FreeHi-C simulation workflow. c. Hierarchical clustering of the original $\mathrm{Hi}-\mathrm{C}$ biological replicates and the FreeHi-C simulated replicates for the Rings, Trophozoites, and Schizonts of $P$. falciparum. d. Hierarchical clustering of the FreeHi-C simulated replicates matching the sequencing depth of the original $P$. falciparum Trophozoites stage sample. e. Hierarchical clustering of the downsampled replicates matching the sequencing depth of the original $P$. falciparum Trophozoites stage. f. HiCRep reproducibility of the contact matrices between the biological replicate 6 of GM12878 and corresponding FreeHi-C simulated replicates of different sequencing depths (first panel) as well as other biological replicates (second panel), FreeHi-C simulated biological replicates (third panel) or downsampled biological replicates (fourth panel) matching the sequencing depth of replicate6. g. HiCRep reproducibility of the contact matrices between pairs of biological replicates of GM12878 simulated by FreeHi-C (orange boxes and lines) or downsampled (purple boxes and lines) to $1 / 2 \times$ sequencing depth of replicate6, $1 \times$ sequencing depth of replicate6, and sequencing depths of replicate4, replicate2, and then replicate3, respectively. 
bioRxiv preprint doi: https://doi.org/10.1101/629923; this version posted May 14, 2019. The copyright holder for this preprint (which was not certified by peer review) is the author/funder, who has granted bioRxiv a license to display the preprint in perpetuity. It is made available under aCC-BY-NC-ND 4.0 International license.

Comparing replicates of A549 with replicates of A549

a
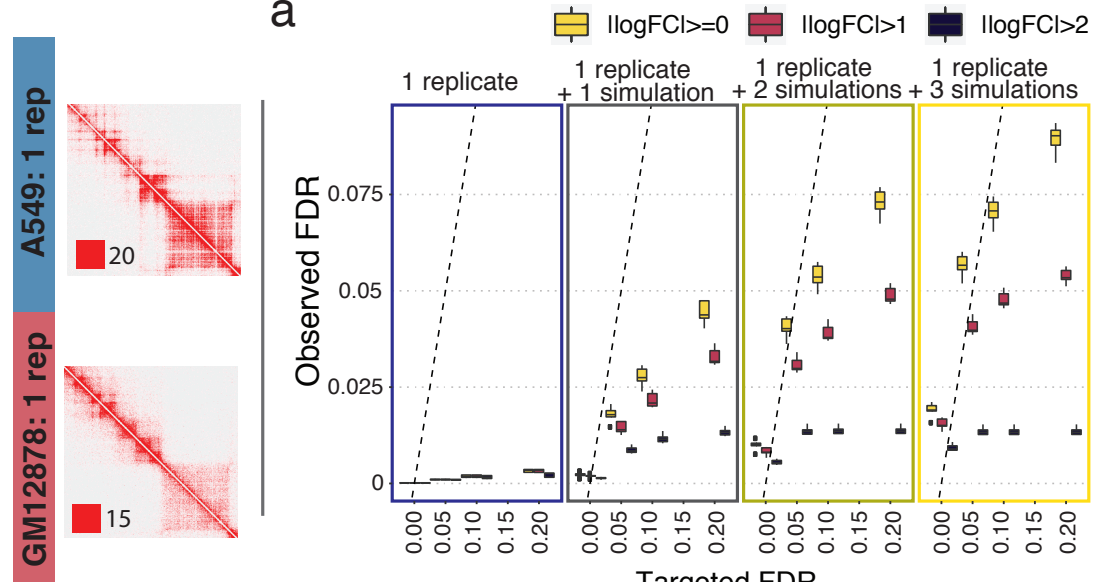

Targeted FDR
Comparing replicates of GM12878 with replicates of A549

b

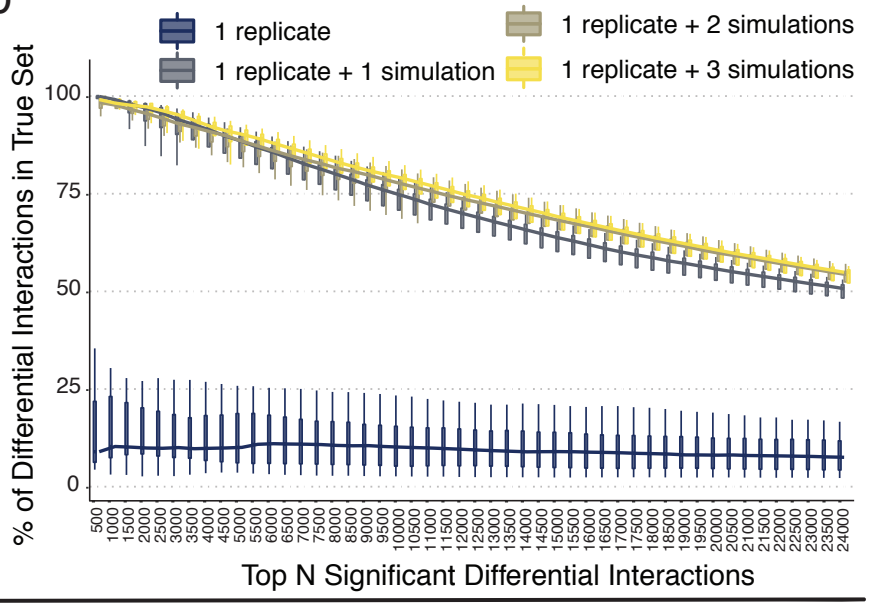

d 1 replicate $\mathrm{GM} 128781$ replicate + simulation vs A549 2 replicates

C

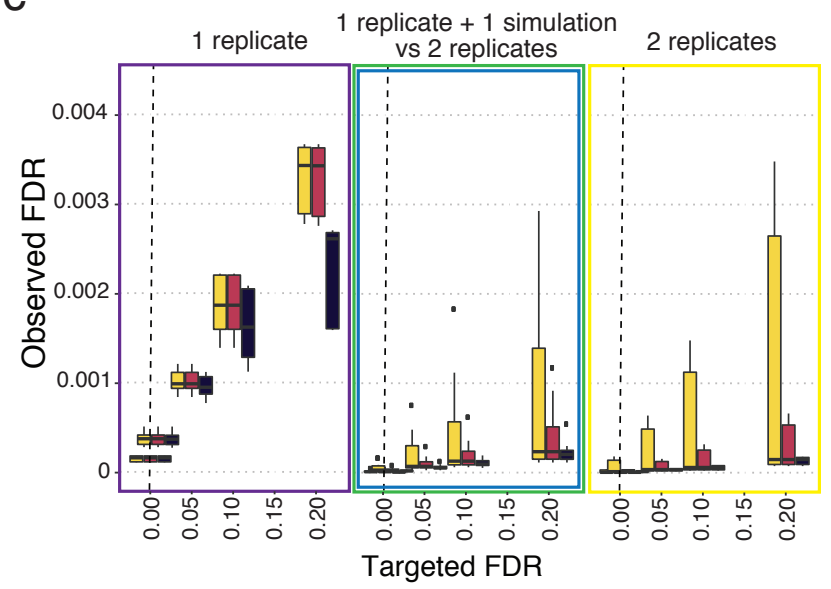

Ф 2 replicates $\mathrm{GM} 128782$ replicates vs A549 1 replicate + simulation

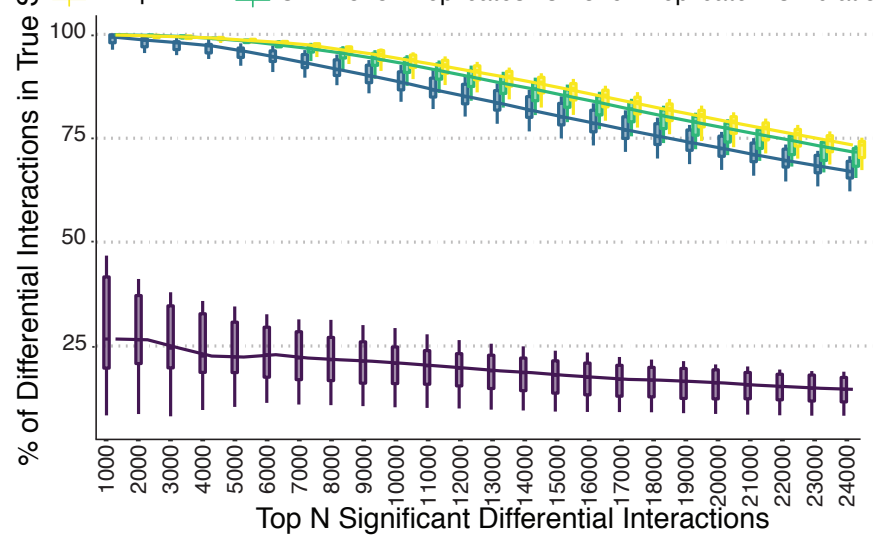

e

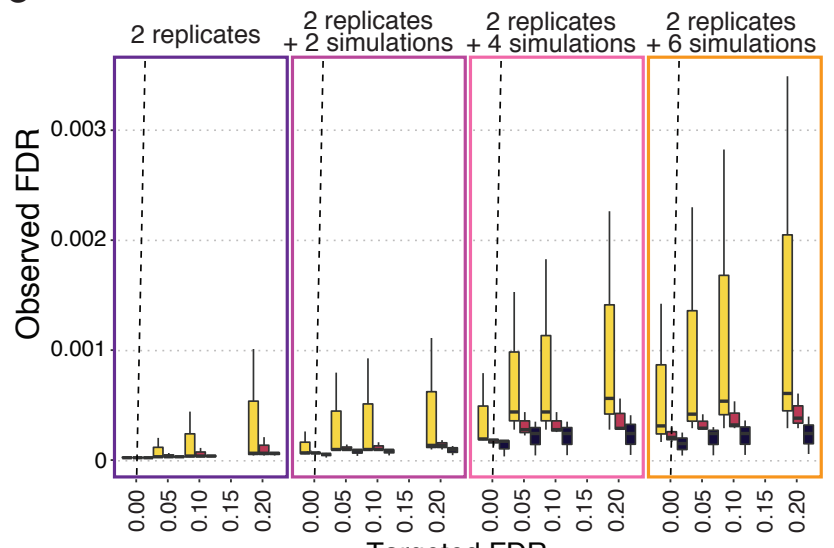

f

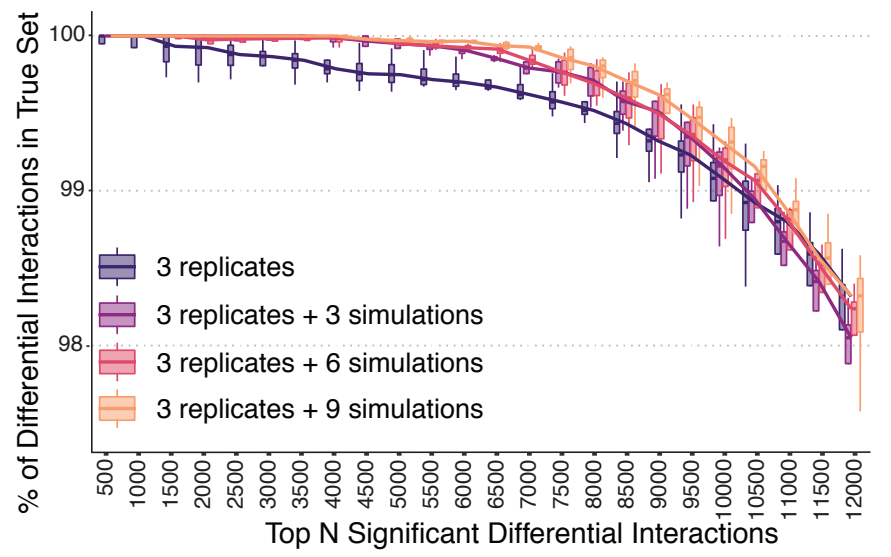


Figure 2 Data augmentation with FreeHi-C simulated replicates improves differential chromatin interactions (DCls) detection. Rows 1 to 3 refer to one replicate per condition (ORPC), uneven numbers of replicates per condition (URPC), and multiple replicates per condition (MRPC) settings, respectively. a, c, and e delineate observed false discovery rates of within-sample comparisons for A549 data (i.e., comparisons of replicate(s) of A549 with other replicate(s) of A549). The dashed lines are $y=x . \mathbf{b}, \mathbf{d}$, and $\mathbf{f}$ display precision, computed as the percentage of top significant DCls of each specific analysis in the "true" differential chromatin interaction list, as a function of top-ranking DCls. The true set is defined by comparing the full set of 4 replicates of GM12878 with 4 replicates of A549. 
bioRxiv preprint doi: https://doi.org/10.1101/629923; this version posted May 14,2019. The copyright holder for this preprint (which was not certified by peer review) is the author/funder, who has granted bioRxiv a license to display the preprint in perpetuity. It is made available under aCC-BY-NC-ND 4.0 International license.

\section{Methods}

\section{FreeHi-C simulation framework}

Processing and training module. FreeHi-C implements the steps outlined in Fig. 11p. It takes as input raw Hi-C sequencing data in the form of FASTQ files, processes the reads, and learns the parameters for the simulation module. The sequence processing module follows a standard protocol ${ }^{24}$ by aligning raw paired-end read files individually and then joining the read ends to form read pairs, followed by interaction validation checking and duplicate removal steps. After obtaining the valid read-pairs (i.e., interactions), it fits an interaction-level mixture model to estimate the genomic fragment interaction frequencies, with the genomic fragments defined by the experimental restriction enzyme cutting sites. This sampling model considers two multinomial distributions with parameters $\overrightarrow{\boldsymbol{\pi}_{0}}=\left\{\pi_{i j}^{0}\right\}$ and $\overrightarrow{\boldsymbol{\pi}_{1}}=\left\{\pi_{i j}^{1}\right\}, i, j=1, \cdots, L$, where $L$ denotes total number of genomic fragments, for generating interaction events. The distribution indexed by the parameter $\pi_{0}$ reflects background interactions, driven by experimental artifacts such as genomic distance whereas the second distribution, $\pi_{1}$, characterizes true biological interaction signals. For each genome fragment "interaction" event, one interaction is drawn from each of the multinomial distributions, and one of them is recorded as the actual interaction event with probability $\alpha$. Consequently, the distribution specified by this interaction-level sampling model is multinomial with vector-valued parameter $\alpha \overrightarrow{\boldsymbol{\pi}_{\mathbf{0}}}+(1-\alpha) \overrightarrow{\boldsymbol{\pi}_{\mathbf{1}}}$. This intuitive sampling model, estimation of which does not require deconvolution of the two distributions, is key for FreeHi-C's successful capture of the interactions in a given biological sample. Once the genomic fragment interaction is sampled, FreeHi-C generates a read pair from this interaction by taking into account strand configuration of the ends of the read pair, mismatch(es), insertion(s), deletion(s), the proportion of chimeric reads, and base quality scores of the reads. Hence, as part of the training module, FreeHi-C estimates these set of parameters empirically from the collection of valid reads. Specifically, FreeHi-C estimates the frequency distributions of numbers of insertions, deletions, and different types of mismatches across all the valid read pairs. Additionally, FreeHi-C processing and training module records the proportion of chimeric reads, i.e., reads pairs where one or both of the read ends are sequenced over the ligation junction, that are rescuable with the aim of preserving this proportion for the simulated reads. Finally, FreeHi-C empirically estimates the distribution of base quality scores for each locus of the Hi-C reads and uses these estimates to ensure that the simulated reads have similar base quality scores as the seed biological replicate.

Simulation module. FreeHi-C simulates fragment pairs from the estimated interaction-level mixture model as genomic fragments that form crosslinks in the $\mathrm{Hi}-\mathrm{C}$ experiment protocol ${ }^{3}$. Ligation procedure in the experiment leads to two fragment junction sites. FreeHi-C randomly generates one of these which is then passed onto the next step to emulate DNA shearing. Two DNA shearing loci are randomly selected within $\pm 500 \mathrm{bp}$, by default, of the selected ligation site. These two shearing loci also work as the starting points of the sequencing procedure. FreeHi-C extracts the sequences of the given length, for example, 36bp for 36bp paired-end sequencing, from these loci and assigns strand direction accordingly. During this sequencing 
step, reads closer than the requested read length to the ligation sites can be generated, as an emulation of chimeric reads. The final step is to introduce noise to the read sequences so that the mismatches and indels match to those in the reads of the original biological sample. Utilizing the empirical distribution of the sequence base quality scores across individual locus, FreeHi-C simulates such scores for each read at the nucleotide level. A key strength of FreeHi-C is that it can generate as many reads as specified by the user and outputs these in the FASTQ format. Furthermore, it processes the resulting reads according to the standard analysis protocol of $\mathrm{Hi}-\mathrm{C}$ reads by the processing module. Through the post-simulation processing, FreeHi-C can directly provide genomics contact counts in a sparse matrix format (BED) compatible with the standard input format of downstream Hi-C analysis. Processing of the raw reads and learning of the parameters can be implemented on individual read pairs followed by a final aggregation; hence this module can be efficiently parallelized. Furthermore, simulations based on the same parameter settings are parallelized at the read-pair generation level.

Simulation parameter settings Different simulation parameter settings lead to variations in sequencing depth and also serve as a user guide for generating simulations that are more close or different than the seed biological replicate. Supplementary Fig. 35 considers a pre-processing parameter (resolution at $10 \mathrm{~kb}$ or $40 \mathrm{~kb}$ ) and four different generative parameters (mutation and indel rates, utilization of chimeric reads, and sequencing depth) that impact the signal to noise ratio of the resulting $\mathrm{Hi}-\mathrm{C}$ dataset. Further systematic evaluation of the reproducibility between simulation samples of varying depths and the seed biological sample emphasizes sequencing depth as the major factor that drives the similarity between simulated $\mathrm{Hi}-\mathrm{C}$ contact matrix and the original one (Supplementary Fig. 36).

\section{Data augmentation with FreeHi-C simulated samples}

When testing for differential chromatin interactions with two or more replicates per condition, we employed a FreeHi-C simulation augmented meta-analysis strategy. This approach generates simulation replicates for each of the original $n$ biological replicates per condition and considers $2^{n}-1$ additional tests to preserve the degrees of freedom of the original test statistic. For example, for a setting with 2 biological replicates per condition, we generated 4 FreeHi-C simulation samples, one per original biological replicate, and evaluated the following comparisons, where $c_{1}$ and $c_{2}$ refer to the two testing conditions under consideration.

$\begin{array}{llllll}\text { Test 1: } & \left(\operatorname{Rep} 1_{c 1, \text { bioSample }},\right. & \left.\operatorname{Rep} 2_{c 1, \text { bioSample }}\right) & \text { vs. } & \left(\operatorname{Rep} 1_{c 2, \text { bioSample }},\right. & \left.\operatorname{Rep} 2_{c 2, \text { bioSample }}\right) \\ \text { Test 2: } & \left(\operatorname{Rep} 1_{c 1, \text { simulation }},\right. & \left.\operatorname{Rep} 2_{c 1, \text { bioSample }}\right) & \text { vs. } & \left(\operatorname{Rep} 1_{c 2, \text { simulation }},\right. & \left.\operatorname{Rep} 2_{c 2, \text { bioSample }}\right) \\ \text { Test 3: } & \left(\operatorname{Rep} 1_{c 1, \text { bioSample }},\right. & \left.\operatorname{Rep} 2_{c 1, \text { simulation }}\right) & \text { vs. } & \left(\operatorname{Rep} 1_{c 2, \text { bioSample }},\right. & \left.\operatorname{Rep} 2_{c 2, \text { simulation }}\right) \\ \text { Test 4: } & \left(\operatorname{Rep} 1_{c 1, \text { simulation }},\right. & \left.\operatorname{Rep} 2_{c 1, \text { simulation }}\right) & \text { vs. } & \left(\operatorname{Rep} 1_{c 2, \text { simulation }},\right. & \left.\operatorname{Rep} 2_{c 2, \text { simulation }}\right)\end{array}$

The p-values of the these tests are then aggregated by Fisher's method ${ }^{\sqrt{28}}$ as $-2 \sum_{i=1}^{M} \log \left(p_{i}\right) \sim$ $\chi_{2 M}^{2}$, where $M=4$ in the above example. Since the tests are not independent, the resulting aggregated p-values are anti-conservative. To dampen this effect, instead of ranking the differential interactions with the Fisher's p-value in the BH procedure ${ }^{27}$, we rank them based on median of their adjusted p-values from individual test. Specifically, instead of ordering the 
hypothesis sequence $H_{(i)}, i=1,2, \ldots, M$, by the aggregated p-values obtained from Fisher's method, we order them by the new significance rank determined by the median adjusted pvalues of the individual tests and denote such ordering as $H_{(r(i))}, i=1,2, \ldots, M$, where $r(i)$ is the index of the interaction that is ranked $i^{\text {th }}$ in the new significant ranking list. Let $k$ be the largest $i$ for which $p_{(r(i))} \leqslant \frac{i}{M} \alpha$, then the $\mathrm{BH}$ procedure rejects all $H_{(r(i))}, i=1,2, \ldots, k$. This is a more conservative procedure than the ordinary BH procedure on the Fisher's p-values because $p_{(i)} \leq p_{(r(i))}$; hence, the number of rejections, $k$, is always smaller or equal to the number of rejections using the BH procedure with Fisher's p-values. The computational experiments support that this reverses the potential anti-conservative effect of aggregating dependent p-values with Fisher's method.

\section{Evaluating the ranking of detected DCIs}

The "true" differential interaction set is approximated by the most significant DCIs detected (FDR $\leq 0.001,0.005,0.01,0.05$, respectively) using 4 biological replicates of GM12878 versus 4 biological replicates of A549. In the sets presented in this paper, we rank the DCIs by their significance order and quantify the fraction of the top $\mathrm{N}$ significant differential interactions that appear in the "true" set where $\mathrm{N}$ varies as 500,1000 , etc. This quantity refers to recovery rate or precision.

Another type of "true" DCI list is specially defined for tests of uneven number of replicates per condition (Supplementary Figs. 21 and 22). In this setting, the true DCI set is defined as the set of most significant interactions in the comparison of rep2 and rep4 of GM12878 with rep1 and rep4 of A549. Accordingly, we measure the precision of the results from the following four comparisons: (i) one replicate out of rep2 and rep4 of GM12878 versus one out of rep1 and rep4 of A459; (ii) one replicate out of rep2 and rep4 of GM12878 with its FreeHi-C augmentation versus one out of rep1 and rep4 of A459 with its FreeHi-C augmentation; (iii) rep2 and rep4 of GM12878 versus one out of rep1 and rep4 of A459 with its FreeHi-C augmentation; (iv) one out of rep2 and rep4 of GM12878 with its FreeHi-C augmentation versus rep1 and rep4 of A459.

\section{Evaluating DCIs detected by FreeHi-C augmentation with RNA-seq and CTCF ChIP-seq}

We evaluated the significance of the observed proportion of differentially expressed (DE) genes between GM12878 and A549 that overlap with significant DCIs using a randomization test. An empirical null distribution for the observed overlap statistics is constructed by randomly selecting an equal number of interactions as significant ones from all valid bin-pairs and overlapping these with the DE genes. The significance level of observed overlap is quantified by the percentage of random selection results that is larger than or equal to the observed statistics. A similar strategy is implemented for evaluating co-localization of DCIs with differential CTCF ChIP-seq peaks.

\section{Data availability}

To study the operating features of FreeHi-C, we utilized two publicly available human HiC datasets as examples of large genomes with four replicates, rep2, rep3, rep4, rep6 from GM12878 $8^{3 / 1}$ cell line and another four replicates, rep1-4, from A54929. Raw FASTQ files for 
GM12878 were downloaded from GEO $^{30}$ under the accession GSE63525 and raw sequences for A549 were obtained from the ENCODE portal ${ }^{31}$ (https://www.encodeproject. org/) with accession ENCSR662QKG. For evaluation of FreeHi-C performance on small genomes, we leveraged three different stages of malaria parasite Plasmodium falciparum red blood cell cycles ${ }^{26}$. GM12878 and A549 are both processed at $40 \mathrm{~kb}$ resolution, and $P$. falciparum at $10 \mathrm{~kb}$.

For validating the differential interaction detection with a differential expression analysis, we utilized RNA-seq gene expression data from the ENCODE portal (accession ENCSR000AED for GM12878 and ENCSR000CTM for A549). Similarly, the CTCF ChIP-seq peak signal files were also downloaded from ENCODE under accession ENCSR000DZN for GM12878 and ENCSR000DPF for A549.

\section{Software availability}

FreeHi-C pipeline is implemented in Python with $\mathrm{C}$ accelerated core calculations and it naturally fits in the high-performance computing environments for parallelization. The source code and instructions for running FreeHi-C are publicly available at https://github.com/ keleslab/FreeHiC. 
1. Dekker, J., Rippe, K., Dekker, M., Kleckner, N.: Capturing chromosome conformation. Science 295(5558) (2002) 1306-11

2. Lieberman-Aiden, E., Van Berkum, N.L., Williams, L., Imakaev, M., Ragoczy, T., Telling, A., Amit, I., Lajoie, B.R., Sabo, P.J., Dorschner, M.O., et al.: Comprehensive mapping of long-range interactions reveals folding principles of the human genome. Science 326(5950) (2009) 289-293

3. Rao, S.S.P., Huntley, M.H., Durand, N.C., Stamenova, E.K., Bochkov, I.D., Robinson, J.T., Sanborn, A.L., Machol, I., Omer, A.D., Lander, E.S., Aiden, E.L.: A 3D map of the human genome at kilobase resolution reveals principles of chromatin looping. Cell 159(7) (2014) $1665-1680$

4. Whalen, S., Truty, R.M., Pollard, K.S.: Enhancer-promoter interactions are encoded by complex genomic signatures on looping chromatin. Nature Genetics 48(5) (2016) 488

5. Roy, S., Siahpirani, A.F., Chasman, D., Knaack, S., Ay, F., Stewart, R., Wilson, M., Sridharan, R.: A predictive modeling approach for cell line-specific long-range regulatory interactions. Nucleic Acids Research 44(4) (2016) 1977

6. Hafez, D., Karabacak, A., Krueger, S., Hwang, Y.C., Wang, L.S., Zinzen, R.P., Ohler, U.: Mcenhancer: predicting gene expression via semi-supervised assignment of enhancers to target genes. Genome Biology 18(1) (2017) 199

7. Ay, F., Bailey, T.L., Noble, W.S.: Statistical confidence estimation for Hi-C data reveals regulatory chromatin contacts. Genome Research 24(6) (2014) 999-1011

8. Servant, N., Varoquaux, N., Lajoie, B.R., Viara, E., Chen, C.J., Vert, J.P., Heard, E., Dekker, J., Barillot, E.: HiC-Pro: an optimized and flexible pipeline for Hi-C data processing. Genome Biology 16(1) (2015) 259

9. Lun, A.T., Smyth, G.K.: diffHic: a Bioconductor package to detect differential genomic interactions in Hi-C data. BMC Bioinformatics 16(1) (2015) 258

10. Ay, F., Noble, W.S.: Analysis methods for studying the 3D architecture of the genome. Genome Biology 16(1) (2015) 183

11. Forcato, M., Nicoletti, C., Pal, K., Livi, C.M., Ferrari, F., Bicciato, S.: Comparison of computational methods for Hi-C data analysis. Nature Methods 14(7) (2017) 679

12. Yang, T., Zhang, F., Yardimci, G.G., Song, F., Hardison, R.C., Noble, W.S., Yue, F., Li, Q.: HiCRep: assessing the reproducibility of Hi-C data using a stratum-adjusted correlation coefficient. Genome Research (2017) gr-220640

13. Djekidel, M.N., Chen, Y., Zhang, M.Q.: Find: differential chromatin interactions detection using a spatial poisson process. Genome Research 28(3) (2018) 412-422 
14. Stansfield, J.C., Cresswell, K.G., Vladimirov, V.I., Dozmorov, M.G.: Hiccompare: an r-package for joint normalization and comparison of hi-c datasets. BMC Bioinformatics 19(1) (2018) 279

15. Yardımcı, G.G., Ozadam, H., Sauria, M.E., Ursu, O., Yan, K.K., Yang, T., Chakraborty, A., Kaul, A., Lajoie, B.R., Song, F., et al.: Measuring the reproducibility and quality of hi-c data. Genome Biology 20(1) (2019) 57

16. Stansfield, J.C., Cresswell, K.G., Dozmorov, M.G.: multihiccompare: joint normalization and comparative analysis of complex hi-c experiments. Bioinformatics (2019)

17. Jin, F., Li, Y., Dixon, J.R., Selvaraj, S., Ye, Z., Lee, A.Y., Yen, C.A., Schmitt, A.D., Espinoza, C., Ren, B.: A high-resolution map of three-dimensional chromatin interactome in human cells. Nature 503(7475) (2013) 290

18. Ursu, O., Boley, N., Taranova, M., Wang, Y.R., Yardimci, G.G., Stafford Noble, W., Kundaje, A.: Genomedisco: A concordance score for chromosome conformation capture experiments using random walks on contact map graphs. Bioinformatics 34(16) (2018) $2701-2707$

19. Ardakany, A.R., Ay, F., Lonardi, S.: Selfish: Discovery of differential chromatin interactions via a self-similarity measure. bioRxiv (2019) 540708

20. DeMaere, M.Z., Darling, A.E.: Sim3c: simulation of hi-c and meta3c proximity ligation sequencing technologies. GigaScience 7(2) (2017) gix103

21. Norton, H.K., Emerson, D.J., Huang, H., Kim, J., Titus, K.R., Gu, S., Bassett, D.S., Phillips-Cremins, J.E.: Detecting hierarchical genome folding with network modularity. Nature Methods 15(2) (2018) 119

22. Spielmann, M., Lupiáñez, D.G., Mundlos, S.: Structural variation in the $3 d$ genome. Nature Reviews Genetics (2018) 1

23. Greenwald, W.W., Li, H., Benaglio, P., Jakubosky, D., Matsui, H., Schmitt, A., Selvaraj, S., DAntonio, M., DAntonio-Chronowska, A., Smith, E.N., et al.: Subtle changes in chromatin loop contact propensity are associated with differential gene regulation and expression. Nature Communications 10(1) (2019) 1054

24. Zheng, Y., Ay, F., Keles, S.: Generative modeling of multi-mapping reads with mhi-c advances analysis of hi-c studies. eLife 8 (2019) e38070

25. Durand, N.C., Robinson, J.T., Shamim, M.S., Machol, I., Mesirov, J.P., Lander, E.S., Aiden, E.L.: Juicebox provides a visualization system for Hi-C contact maps with unlimited zoom. Cell Systems 3(1) (2016) 99-101 
26. Ay, F., Bunnik, E.M., Varoquaux, N., Bol, S.M., Prudhomme, J., Vert, J.P., Noble, W.S., Le Roch, K.G.: Three-dimensional modeling of the P. falciparum genome during the erythrocytic cycle reveals a strong connection between genome architecture and gene expression. Genome Research 24(6) (2014) 974-988

27. Benjamini, Y., Hochberg, Y.: Controlling the false discovery rate: a practical and powerful approach to multiple testing. Journal of the Royal Statistical Society: Series B (Methodological) 57(1) (1995) 289-300

28. Fisher, R.A.: Statistical methods for research workers. Genesis Publishing Pvt Ltd (2006)

29. Dixon, J.R., Xu, J., Dileep, V., Zhan, Y., Song, F., Le, V.T., Galip Gr̈kan Yardımc1, A.C., Bann, D.V., Wang, Y., Clark, R., Zhang, L., Yang, H., Liu, T., Iyyanki, S., An, L., Pool, C., Sasaki, T., Rivera-Mulia, J.C., Özadam, H., Lajoie, B.R., Kaul, R., Buckley, M., Lee, K., Diegel, M., Pezic, D., Ernst, C., Hadjur, S., Odom, D.T., Stamatoyannopoulos, J.A., Broach, J.R., Hardison, R.C., Ay, F., Noble, W.S., Dekker, J., Gilbert, D.M., Yue, F.: Integrative detection and analysis of structural variation in cancer genomes. Nature Genetics (September 2018) https://www.nature.com/ articles/s41588-018-0195-8.

30. Barrett, T., Wilhite, S.E., Ledoux, P., Evangelista, C., Kim, I.F., Tomashevsky, M., Marshall, K.A., Phillippy, K.H., Sherman, P.M., Holko, M., et al.: NCBI GEO: archive for functional genomics data setsupdate. Nucleic Acids Research 41(D1) (2012) D991-D995

31. Consortium, E.P., et al.: An integrated encyclopedia of dna elements in the human genome. Nature 489(7414) (2012) 57 


\section{Supplementary Texts}

In addition to studying the reproducibility of Hi-C datasets using HiCRep in a sequencing depth debiased manner, FreeHi-C simulations can also be leveraged to study sequencing depth dependent characteristics of Fit-Hi- $\mathrm{C}^{7]}$ and similar methods in detecting significant interactions. A standard benchmarking approach for methods like Fit-Hi- $\mathrm{C}^{7]}$ is to ask what proportion of the significant interactions are reproducibly recovered among different biological replicates. Similar to the findings of HiCRep reproducibility, the recovery ratio of the significant interactions is higher in the simulated replicates and substantial differences in sequencing depths can make the recovery rate of high depth biological replicates seem close to those of the lower depth simulated replicates of low depth biological replicates (Supplementary Fig. 37). Simulation and downsampling of biological replicates to the sequencing depth of the target replicate enable debiasing of the comparison of significant interaction detection between simulated replicates and biological replicates from sequencing depth (Supplementary Fig. 37b and 38). 